\title{
Review
}

\section{Recent Progress in Mycoplasma pneumoniae Infection}

\author{
Anqiao Zhong \\ Department of Respiratory Medicine, Yidu Central Hospital Affiliated to Weifang Medical College, Qingzhou, China
}

\author{
Keywords \\ Mycoplasma pneumoniae; immune response; \\ drug resistance; summary \\ Correspondence \\ Anqiao Zhong, \\ E-mail: zhongaq@126.com
}

DOI: 10.1515/ii-2017-0102

\begin{abstract}
Mycoplasma pneumoniae ( $\mathrm{Mp}$ ) is a definite respiratory pathogen affecting people of all ages. This organism is adsorbed on host cell surface through extreme adherent organelles, generating peroxide ions and possible exotoxins. Mp may directly invade host cells and cause latent infections. Induced immunoreactive injury is one of the main factors resulting in clinical symptoms of Mp infection. Polymerase chain reaction (PCR) and serological detection should be combined for diagnosis of $\mathrm{Mp}$ infection when nasopharyngeal and oropharyngeal specimens are simultaneously obtained and detected by PCR. The most reliable basis for diagnosis can be obtained with two serological tests in different courses of the disease. Culture methods also bear significance in diagnosing Mp infections and in vitro drug sensitivity tests.
\end{abstract}

Currently, more than 200 species of Mycoplasma are known. However, only several are pathogenic for humans. Among these species, Mycoplasma pneumoniae (Mp) is the most studied. However, further explorations are required to determine the manner of Mp interaction with host cells, its effects on the immune system, and extrapulmonary diseases that it causes. Currently, Mp acts as a definite respiratory pathogen affecting people in all age groups, and its compatibility with host cells is considered the main virulence factor. To date, controversies surround whether Mp can parasitize cells and cause latent infection. Autoimmune and extrapulmonary expression rates induced by $\mathrm{Mp}$ infection reaches up to $25 \%$ in infected populations ${ }^{[1]}$. Mp lacks standardized in vitro drug-sensitivity test. Currently, multiple investigation reports are available on mutations of macrolide drug-resistance gene. Compared with macrolidesensitive Mp strain infections, people infected with drugresistant $\mathrm{Mp}$ strain present significantly prolonged stage of effervescence. Mp infection outbreaks occur every four to seven years; inactivated $\mathrm{Mp}$ vaccine can reduce $40 \%$ of $\mathrm{Mp}$ related respiratory tract infections ${ }^{[2]}$.

\section{Mp pathogenic cell and molecular basis}

Interaction between $\mathrm{Mp}$ and host cell is achieved by an adsorbed organelle, which comprises $\mathrm{P} 1$ protein containing $170 \mathrm{ku}$, high molecular weight (HMW) 1, 2, and 3, and proteins P90, P40, and P30. HMW is responsible for formation and maintenance of adhesive structure with extreme protrusions, enabling the entire $\mathrm{Mp}$ to form flask-like extensions. Proteins B, C, and P1 form adhesive complex and bind with host cell receptors, such as sialic acid glycosyl conjugates, glycoproteins, and sulfated glycolipids. Successful adherence to respiratory tract epithelium of host is a prerequisite for $\mathrm{Mp}$-induced infection. Epidemiological studies showed that P1 of Mp features at least two major genotypes, and changes in P1 may be related with generation of genotype-specific antibodies, short duration of immunity, and clinical recurrence of infections. Successfully adhered Mp produces superoxide anions, making host cells susceptible to peroxide damage by inhibiting host catalase. $\mathrm{HPr}$ kinase plays a major regulatory role in $\mathrm{Mp}$ infection. Mp degrades phospholipids in host cell membrane to obtain glycerol. Glycerol activates HPr kinase to activate $\mathrm{HPr}$ protein, thereby producing peroxide and superoxide anions and subsequently leading to host cell damage, which is mainly manifested as disappearance of respiratory epithelial cell cilia, vacuolization, decreased sugar utilization, amino acid uptake, and synthetic ability manifested as persistent dry cough in clinical practice. Mp may become pathogenic by synthesizing and releasing exotoxins ${ }^{[3]}$. Mp genome- 
wide scans showed a protein that is highly homologous to diphtheria bacillus toxin subunit S1, and it specifically binds to surface-active protein A. Synthetic protein function demonstrates that this molecule exhibits adenosine diphosphate ribosyltransferase activity with potential exotoxin effects. Thus, the protein was named communityacquired respiratory distress syndrome toxin. However, host cell receptor of this protein remains undiscovered, and in terms of toxin function, the role of its specific properties in binding with surface-active protein A remains unclear. Mp does not invade host cells, but genomic scans indicated that Mp lacks some genes that are essential for independent living; this condition is similar to that of strict intracellular parasites ${ }^{[4]}$. Although laboratory analysis showed Mp growth and reproduction in cells, and clinical findings also revealed $\mathrm{Mp}$ chronic or latent infections, requirement of long-term treatment, and other phenomena associated with intracellular parasite infections, further studies are required to clarify mechanism of $\mathrm{Mp}$ parasitism in natural circumstances.

\section{Congenital and adaptive immune response}

When Mp reaches the lower respiratory tract, it is phagocytosed by activated macrophages through antibody and complement conditioning. Neutrophils and lymphocytes also show chemotaxis toward infected sites. Duration of immune response induced by $\mathrm{Mp}$ infection is short, and secondary infection is very common. Some individuals become long-term carriers. Considering that congenital hypoclobulinemia patients are prone to chronic mycoplasma respiratory tract infections or extrapulmonary mycoplasma-disseminated infections, humoral immunity bears significance in controlling Mp. Through P1 adhesion unit, $\mathrm{Mp}$ binds to sialic acid receptor in mastocyte membrane and induces mastocytes to generate interleukin (IL)-4 ${ }^{[5]}$. $\mathrm{Mp}$-derived lipoproteins can bind to Toll-like receptor on macrophage membrane and activate macrophages to generate tumor necrosis factor $\alpha$, IL-8, and IL-1 $\beta$. Intensified cellmediated immune response results in increased production of cytokines, more serious clinical symptoms of lung injury, and possible autoimmune response. Up to $25 \%$ of $\mathrm{Mp}$ infected persons may develop extraperitoneal manifestations because of autoimmune reactions, which include the skin, nerves, cardiovascular system, kidney, gastrointestinal tract, skeletal muscle, and hematopoietic systems. Neurological complications are the most common as Mp cell membrane contains several glycolipids and liposaccharide antigens, which can form cross antigens with neuronal components. Antiganglioside GM1 antibody and galactose brain glycoside antibody were detected in Mp-related encephalitis ${ }^{[6]}$, along with increased cerebrospinal fluid cytokines IL-6 and IL-8 . Mp nucleic acid was detected by nucleic acid hybridization in brain tissues. Mp antigen was immunohistochemically detected in brain tissues. Mp was detected in cerebrospinal fluid using polymerase chain reaction (PCR) and culture [7]; all results suggest that Mp not only can pass through immune-damaged cells but also spread infection to the central nervous system. Evidence of Mp infection was reported in PCR or culture in blood, synovial fluid, pericardial fluid, and skin lesions. Thus, immune damage and direct invasion of Mp may cause extrapulmonary manifestations of Mp infection.

\section{Function of Mp in asthma and chronic lung disease}

Mp infection is sometimes difficult to treat and causes chronic clinical processes that play certain roles in adult rheumatoid arthritis, adolescent idiopathic arthritis, Crohn' $\mathrm{s}$ disease, and asthma, because of the presence of host cell infections, immunoregulatory effects, and surface antigen variations ${ }^{[8]}$. Compared with healthy controls, asthmatic people present significantly higher detection rate of $\mathrm{Mp}$, whereas macrolide antibiotics significantly improve lung function in $\mathrm{Mp}$-infected asthma patients; enduring $\mathrm{Mp}$ infection was observed during long-term monitoring of children with impaired respiratory function; experiments also showed that Mp-induced inflammatory mediators, such as IgE, substance $P$, neurokinin 1 , and IL-5, can aggravate asthma; all these observations indicate that $\mathrm{Mp}$ is pathogenic in asthma ${ }^{[9]}$.

\section{Mp laboratory testing}

Mp culture identification requires up to six weeks. Serological experiments aid in crowd epidemiological investigation. However, such methods feature weak reliability for individual diagnosis. Thus, effective clinical diagnosis of $\mathrm{Mp}$ remains inadequate. Specific antibodies are detected within one week after Mp infection, peak within three to six weeks, and then reduce afterward. Increase in serum antibodies in acute and recovery stages is reliable for diagnosis of $\mathrm{Mp}$ 
infection. However, this variable cannot be used for early diagnosis. IgM or IgA detection aids early diagnosis, but a positive proportion exists among healthy people. She et al. ${ }^{[10]}$ performed investigations on culture of 24,677 cases of respiratory tract Mp specimens, among which 10 cases were positive (0.04\%). PCR results of 8,509 respiratory tract Mp specimens showed 167 positive cases (1.9\%). IgM antibody detection of 92,507 serum Mp specimens detected 6,049 positive cases (6.6\%). Considering low Mp culture rate of clinical specimens, PCR combined with serological detection may serve as feasible diagnostic method. Nasopharyngeal and oropharyngeal specimens simultaneously obtained for PCR may provide reliable basis for diagnosis. For sputum patients, positive rate of PCR in sputum samples is higher than that in nasopharyngeal specimens and can well reflect infections in the lower respiratory tract ${ }^{[11]}$. Although culture method features poor sensitivity, its manifests excellent specificity. Especially for Mp infections manifested outside the lungs, culture positivity is the best evidence for direct invasion of Mp. Clinical isolates can also be used for further genotyping and drug resistance analysis. Gnarpe et al. ${ }^{[12]}$ observed that pharyngeal carriage rate of $\mathrm{Mp}$ reaches $4.6 \%-13.5 \%$ in healthy people. Thus, for patients who test positive in PCR and negative in serology, asymptomatic carrier, antibodies not produced by immune deficiency, or early successful inhibition of Mp by antibiotics should be considered. For patients who test negative in PCR and positive in serology, existence of substances contained in specimen and inhibits PCR material or immune memory should be considered.

\section{Mp macrolide antibiotic resistance and drug-sensitivity test}

Under normal circumstances, $\mathrm{Mp}$ is sensitive to macrolides, tetracyclines, and quinolones antibiotics. In cases of mutations in Mp 23S rRNA peptide transferase-binding region, macrolide exhibits decreased binding ability to $\mathrm{Mp}$ ribosome. Reported Mp 23S rRNA point mutations include A2063G, A2064G, and C785T. Liu et al. ${ }^{[13]}$ reported that among 55 cases of clinical isolates in Shanghai, 44 cases (83\%) were resistant to macrolide, and all resistant strains showed A2063G 23SrRNA mutation. Xin et al. ${ }^{[14]}$ reported that among 50 cases of clinical isolates, 46 cases (92\%) were resistant to macrolide; among these cases, 40 contained $23 \mathrm{~S}$ rRNA A2063G; one case featured A2063C, and five cases presented A2064G. Suzuki et al. ${ }^{[15]}$ reported that compared with macrolide-sensitive Mp strains, stage of effervescence was significantly prolonged in people infected with drugresistant Mp strains. However, no serious outcomes, such as respiratory failure, occurred, possibly because pathogenicity of $\mathrm{Mp}$ is based on immune injury, and macrolide drugs posed immunomodulating effects. Miyashita et al. ${ }^{[16]}$ reported two cases of adult macrolide-resistant $\mathrm{Mp}$-induced pneumonia, and Lung et al. ${ }^{[17]}$ reported one case of severe macrocytosis caused by macrolide-resistant $\mathrm{Mp}$ in children. As tetracycline is an antimicrobial agent, and wide use of quinolones may probably result in resistant strains with single-point mutations, and as Mp infection occurs mostly in children, macrolide drugs are still the first choice of treatment. Currently, clinical practice requires development of reliable and reproducible Mp drug-susceptibility test. However, no standardized $\mathrm{Mp}$ in vitro susceptibility test is available because of long incubation period and harsh cultivation conditions for $\mathrm{Mp}$ and lack of definite standard test strains for minimal inhibitory concentrations.

\section{Summary}

In the future, studies on Mp infection may focus on the following aspects: development of standardized $\mathrm{Mp}$ in vitro culture and drug-sensitivity test, discovery of new $\mathrm{Mp}$ virulence factors through genome scanning and protein identification, and exploration of congenital and adaptive immunity of hosts to Mp infection.

\section{Declarations}

\section{Acknowledgements}

No.

\section{Competing interests}

The author declare that he has no competing interest.

\section{Authors' contributions}

AQ Zhong made the literature analysis and wrote, discussed and revised the manuscript of this review.

\section{References}

1 Waites KB, Talkington DF. Mycoplasma pneumoniae and its role as a human pathogen. Clin Microbiol Rev, 2004, 17(4):697-728.

2 Linchevski I, Klement E,Nir-Paz R.Mycoplasma pneumoniae vaccine protective efficacy and adverse reactions-Systematic review and metaanalysis.Vaccine, 2009, 27(18):2437-46. 
3 Kannan TR, Provenzano D, Wright JR, et al. Identification and characterization of human surfactant protein A binding protein of Mycoplasma pneumoniae. Infect Immun, 2005, 73(5): 2828-34.

4 Meseguer MA, Alvarez A, Rejas MT, et al. Mycoplasma pneumoniae: a reduced-genome intracellular bacterial pathogen. Infect Genet Evol, 2003, 3(1):47-55.

5 Luo D, Dai Y, Duffy LB, et al. Inhibition of message for FceRI a chain blocks mast cell IL-4production induced by co-culture with Mycoplasma pneumoniae. Microb Pathog, 2008, 44(4):286.

6 Christie LJ, Honarmand S, Talkington DF, et al. Pediatric encephalitis: what is the role of Mycoplasma pneumonia. Pediatrics, 2007, 120(2):305-13.

7 Stamm B, Moschopulos M, Hungerbuehler H, et al. Neuroinvasion by Mycoplasma pneumoniae in acute disseminated encephalomyelitis. Emerg Infect Dis, 2008, 14(4):641-3.

8 Waites KB, Balsih MF, Atkinson TP. New insights into the pathogenesis and detection of Mycoplasma pneumoniae infections. Future Microbiol, $2008,3(6): 635-48$

9 Hong SJ. The Role of Mycoplasma pneumoniae Infection in Asthma. Allergy Asthma Immunol Res, 2012, 4(2):59-61.

10 She RC, Thurber A, Hymas WC, et al. Limited utility of culture for Mycoplasma pneumoniae and Chlamydophila pneumoniae for diagnosis of respiratory tract infections. J Clin Microbiol, 2010, 48(9):3380-2.
11 Cho MC, Kim H, An D, et al. Comparison of sputum and nasopharyngeal swab specimens for molecular diagnosis of Mycoplasma pneumoniae,Chlamydophila pneumoniae, and Legionella pneumophila[J].Ann Lab Med,2012,32(2):133-8.

12 Gnarpe J,Lundback A,Sundelof B, et al.Prevalence of Mycoplasma pneumoniae in subjectively healthy individuals. Scand J Infect Dis, 1992, 24(2):161-4.

13 Liu Y, Ye X, Zhang H, et al. Antimicrobial susceptibility of Mycoplasma pneumoniae isolates and molecular analysis of macrolideresistant strains from Shanghai,China. Antimicrob Agents Chemother, 2009, 53(5):2160-2

14 Xin D, Mi Z, Han X, et al. Molecular mechanisms of macrolide resistance in clinical isolates of Mycoplasma pneumoniae from China. Antimicrob Agents Chemother, 2009, 53(5):2158-9.

15 Suzuki S, Yamazaki T, Narita M, et al. Clinical evaluation of macrolideresistant Mycoplasma pneumoniae. Antimicrob Agents Chemother, 2006, 50(2):709-12.

16 Miyashita N, Maruyama T, Kobayashi T, et al. Community-acquired macrolide-resistant Mycoplasma pneumoniae pneumonia in patients more than 18years of age. J Infect Chemother, 2011, 17(1):114-8.

17 Lung DC,Chan YH,Kwong L, et al.Severe community-acquired pneumonia caused by macrolide-resistant Mycoplasma pneumoniae in a 6-year-old boy. Hong Kong Med J, 2011, 17(5):407-9. 